\title{
Secondary intervention with aminoguanidine retards the progression of diabetic retinopathy in the rat model
}

\author{
H.-P. Hammes ${ }^{1}$, D. Strödter ${ }^{1}$, A. Weiss ${ }^{1}$, R. G. Bretzel ${ }^{1}$, K.Federlin ${ }^{1}$, M. Brownlee ${ }^{2}$ \\ 1 Third Medical Department, Justus-Liebig-University, Giessen, Germany \\ ${ }^{2}$ Diabetes Research Center and Departments of Medicine and Pathology, Albert Einstein College of Medicine, Bronx, New York, \\ USA
}

Summary Primary prevention with aminoguanidine an inhibitor of advanced glycation end product (AGE) formation - has been successfully employed to prevent diabetic retinopathy in the rat. However, it is unknown whether inhibition of AGE formation is still effective in a secondary intervention strategy. The present study addresses this question by comparing secondary intervention with aminoguanidine with syngeneic islet transplantation in the rat model. After 6 months of diabetes, one group was treated with aminoguanidine $(50 \mathrm{mg} / 100 \mathrm{ml}$ drinking water; $\mathrm{D}-\mathrm{AG}$ ) while another group received syngeneic transplantation of collagenase-ficoll isolated islets by intraportal injection (Tx). After an additional 4 months, both groups were compared to a normal (NC 10) and diabetic (DC 10) control group. Retinal autofluorescence was increased 2.5-fold after 6 months and increased 3.7-fold after 10 months of diabetes $(p<0.001)$. Aminoguanidine and islet Tx retarded the further accumulation of autofluorescence equally ( $p<0.001$ vs DC 10 ), although the values were higher than those observed in DC at 6 months $(p<0.001)$. Diabetes was associated with a 2.7 -fold increase in acellular capillaries after 6 months and a 4.1-fold increase after 10 months. Treatment with aminoguanidine or islet Tx reduced but did not completely attenuate the progression of vascular occlusion $(p<0.001$ vs DC $10 ; \mathrm{D}-\mathrm{AG}$ vs DC $6, p<0.05$; Tx vs DC $6, p<0.01)$. Both treatments reduced endothelial proliferation $(22.4 \%$ after 10 months; $p<0.001$ ) and completely arrested pericyte dropout (40\% after 10 months; $p<0.001$ ).

These data demonstrate that aminoguanidine is as effective as islet transplantation in retarding the progression of diabetic retinopathy in a secondary prevention setting. [Diabetologia (1995) 38: 656-660]

Key words Diabetic retinopathy, rat model, aminoguanidine, glycation, secondary intervention, islet transplantation.
Diabetic retinopathy affects almost every insulin-dependent diabetic (IDDM) patient and a majority of patients with non-insulin-dependent diabetes mellitus (NIDDM) with a disease duration of more than 15 years $[1,2]$. Background retinopathy leads to the

Received: 29 August 1994 and in final revised form: 20 December 1994

Corresponding author: Dr. H.-P. Hammes, Third Medical Department, Justus-Liebig-University, Rodthohl 6, D-35385 Giessen, Germany

Abbreviations: Tx, Islet transplantation; AGE, advanced glycation end product; iNOS, inducible form of nitric oxide synthase. development of proliferations in $60 \%$ of IDDM patients [3] which is the major cause of blindness in the adult population of western countries [4]. Experimental and clinical studies have demonstrated the causal relationship between the cumulative history of glycaemia and the development of retinopathy [5, 6]. Animal studies using aminoguanidine - an inhibitor of hyperglycaemia-derived advanced glycation end product (AGE) formation - have shown that experimental diabetic retinopathy can be prevented by the inhibition of AGE formation [7,8]. However, it is not known whether aminoguanidine would be effective in a secondary strategy. We studied this question in an established rat model of experimental diabetic retinopathy in comparison to secondary res- 
toration of near-normoglycaemia by islet transplantation, and found secondary treatment with aminoguanidine to be as effective as islet transplantation in retarding the progression of experimental diabetic retinopathy.

\section{Materials and methods}

Animals. Male Lewis rats (Han.Lew, Zentralinstitut für Versuchstierkunde, Hannover, Germany) weighing 200-250 g at the outset were used in this experiment. Diabetes was induced by i.v. injection of streptozotocin (STZ, $65 \mathrm{mg} / \mathrm{kg}$ body weight; Boehringer Mannheim, Mannheim, Germany). Only animals with blood glucose exceeding $15 \mathrm{mmol} / \mathrm{l}$ at 1 week after STZinjection were included into the diabetic groups. All rats were fed a regular laboratory rodent chow (Altromin, Lage, Germany) ad libitum and had free access to drinking water.

Blood glucose (glucose oxidase method) and body weight were monitored at regular intervals. After 6 months of constant hyperglycaemia, animals were randomly assigned to receive either $50 \mathrm{mg} / 100 \mathrm{ml}$ drinking water aminoguandineHCL (Riedel-de Haen, Hannover, Germany) (D-AG) or intraportal islet transplantation ( $\mathrm{Tx}$ ) (see below) or no treatment (DC). A non-diabetic (NC 6) and diabetic (DC 6) group were killed and the eyes removed for examination. Water consumption was identical in the aminoguanidine-treated and the untreated diabetic group.

Glycated haemoglobin (Glyc Affin GHb, Isolab, DRG, Marburg, Germany) was measured in each group at the time of killing.

Islet transplantation. Islet transplantation was performed using the method of Lacy and Kostianovsky [9]. Briefly, the donor pancreas (non-diabetic Lewis rat) was digested with $10 \mathrm{ml}$ collagenase $(0.02 \%)$ via the ductus choledochus, separated from the surrounding small gut and incubated for $45 \mathrm{~min}$ at $37^{\circ} \mathrm{C}$. Before centrifugation ( $30 \mathrm{~min}, 900 \mathrm{~g}$ ) against a Ficoll gradient (Sigma, Heidelberg, Germany), the digest was homogenized by whirling on a vortex for $15 \mathrm{~s}$ and washed twice in ice-cold Hanks' solution (Gibco, Eckenstein, Germany). A total of approximately 1000 freshly isolated islets was given intraportally in the anaesthesized animal.

After 10 months (i.e. after 4 months of treatment), untreated diabetic rats (DC 10), their age-matched non-diabetic counterparts (NC 10) and diabetic rats treated with aminoguanidine (D-AG) or islet transplantation ( $\mathrm{Tx}$ ) were killed.

The time course of the study is given in Figure 1.

Retinal digest preparations. Retinae were obtained at the time points indicated above after enucleation of the eyes from the animals under deep anaesthesia and immediately fixed in $4 \%$ buffered formalin.

Retinal vascular preparations were performed using a pepsin-trypsin digestion technique as previously described $[7,10]$. Briefly, a combined pepsin (5\% pepsin in $0.2 \%$ hydrochloric acid for $1.5 \mathrm{~h})$ trypsin $(2.5 \%$ in $0.2 \mathrm{~mol} / \mathrm{l}$ Tris for $15-30 \mathrm{~min}) \mathrm{di-}$ gestion was used to isolate the retinal vasculature and the specimens were stained with periodic acid Schiff. Retinal autofluorescence was measured in unstained digest preparations according to a previously published method $[7,8]$.

Acellular capillaries were quantitated by a modification of the method of Engerman and Kern [11]. Using a grid of 100 fields, 10 microscopic fields covering a total area of $6.76 \mathrm{~mm}^{2}$ of retinal area were scored for the presence of acellular occluded vessels (integration ocular Olympus/400×). Each field con-

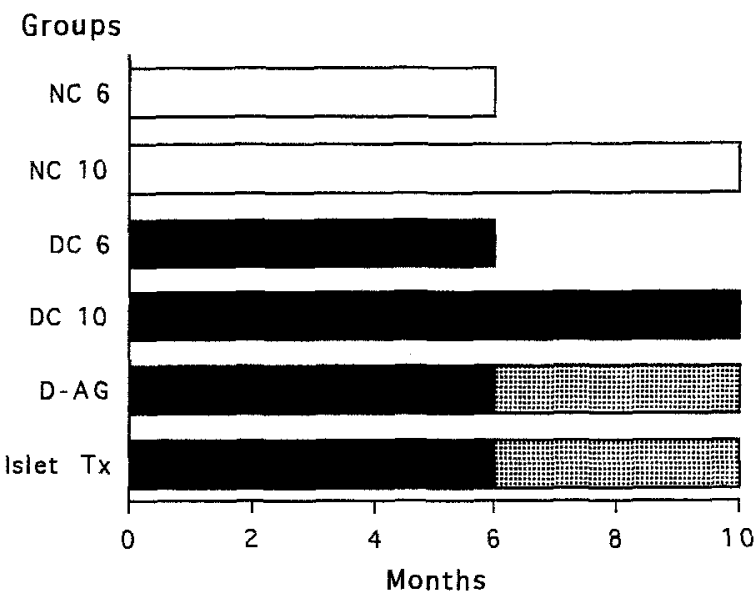

Fig. 1. Schematic of the study design. Non-diabetic $(\square)$ and diabetic rats $(\square$ ) were killed after 6 and 10 months respectively. Treatments with aminoguanidine (D-AG) and islet transplantation (Islet $T_{x}$ ) were started after 6 months of diabetes

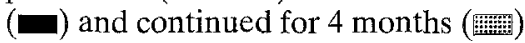

taining acellular capillary segments was recorded as positive, and values were normalized to $\mathrm{mm}^{2}$ of retinal area.

The total number of endothelial cells and pericytes (average approximately 170 cells per retina) was counted in 10 randomly selected fields of the retina using an image analysing system (CUE 2; Olympus Opt. Inc., Hamburg, Germany). Differentiation of pericytes from endothelial cells was performed according to the criteria of Kuwabara and Cogan [10]. Pericyte identification was facilitated using a polyclonal anti-vitronectin antibody (rb 69, kindly provided by Klaus Preissner, Max-Planck-Institut, Bad Nauheim, Germany). The numbers of each cell type were normalized to the relative capillary density (numbers of cells per $\mathrm{mm}^{2}$ of capillary area).

One retina per animal was used for morphometric evaluation. Morphometry was performed with the identity of the samples remaining unknown.

\section{Statistical analysis}

All parameters are given as mean \pm SD unless otherwise stated. One-way analysis of variance was performed, followed by Bonferroni- $t$-tests to correct for multiple comparisons and assign differences to individual groups where overall significance $(p<0.05)$ was attained.

\section{Results}

STZ-induced diabetes resulted in stable hyperglycaemia (Fig. 2) and progressive weight loss as well as in an almost threefold increase in Amadori product formation (Table 1). Aminoguanidine that was given from month 6 to month 10 (D-AG) did not alter the level of Amadori products $\left(\mathrm{HbA}_{1}\right)$. Plasma aminoguanidine levels were $18.64 \pm 2.98 \mu \mathrm{g} / \mathrm{ml}$ in the DAG group. Islet transplantation, performed after 6 months of diabetes, was accompanied by significantly improved glucose and Amadori product levels (blood glucose and $\mathrm{HbA}_{1}$ Tx vs DC $10 p<0.001$, re- 
Table 1. Body weight and glycaemic control of non-diabetic, diabetic and treated diabetic rats

\begin{tabular}{lrlcl}
\hline & $n$ & $\begin{array}{l}\text { body weight } \\
(\mathrm{g})\end{array}$ & $\begin{array}{l}\mathrm{HbA}_{1} \\
(\%)\end{array}$ & $\begin{array}{l}\text { Aminoguanidine } \\
(\mu \mathrm{g} / \mathrm{ml})\end{array}$ \\
\hline NC 6 & 7 & $390 \pm 39$ & $5.3 \pm 0.50$ & - \\
NC 10 & 6 & $494 \pm 48^{\mathrm{a}}$ & $5.3 \pm 0.52$ & - \\
DC 6 & 11 & $275 \pm 21^{\mathrm{a}}$ & $13.5 \pm 1.56^{\mathrm{a}}$ & - \\
DC 10 & 6 & $255 \pm 35^{\mathrm{b}}$ & $15.0 \pm 1.70^{\mathrm{b}}$ & - \\
D-AG & 6 & $204 \pm 16^{\mathrm{b}}$ & $14.6 \pm 0.85^{\mathrm{b}}$ & $18.64 \pm 2.98$ \\
Tx & 11 & $405 \pm 34^{\mathrm{b}, \mathrm{d}}$ & $6.7 \pm 1.10^{\mathrm{c}, \mathrm{d}, \mathrm{c}}$ & - \\
\hline
\end{tabular}

NC 6, non-diabetic after 6 months; NC 10, non-diabetic after 10 months; DC 6 , diabetic after 6 months; DC 10 , diabetic after 10 months; D-AG, diabetic rats, treated with aminoguanidine; $\mathrm{Tx}$, diabetic rats, treated with islet transplantation. Results are shown as mean $\pm \mathrm{SD} .{ }^{\mathrm{a}} p<0.001$ vs NC $6 ;{ }^{\mathrm{b}} p<0.001$ vs NC $10 ;{ }^{c} p<0.01$ vs NC $10 ;{ }^{d} p<0.001$ vs DC $10 ;{ }^{\circ} p<0.05$ vs NC 10. Differences of $\mathrm{HbA}_{1}$ between DC 6, DC 10 and DAG were not significant. Plasma aminoguanidine concentrations were measured according to the method of Beaven et al. [12]

spectively) and a significant weight gain (Tx vs DC 10 $p<0.001$ ), although not completely normalized $\left(\mathrm{HbA}_{1} \mathrm{Tx}\right.$ vs NC $10 p<0.05$; blood glucose and body weight Tx vs NC $10 p<0.01$, respectively).

Increased retinal vascular autofluorescence was predominantly observed at the branching sites of precapillary arterioles of diabetic animals and, to some extent, in treated diabetic rats (Fig. 3). The location was identical to those published previously [7].

Autofluorescence quantitation revealed an almost 2.5-fold increase of fluorescent AGEs in DC 6 and a further rise after 10 months of untreated diabetes. Secondary intervention with aminoguanidine largely attenuated the further accumulation of fluorescent AGEs in the retina (DC $10641 \pm 20 \mathrm{AU}$ vs D-AG $467 \pm 8 ; p<0.001)$. Secondary islet transplantation reduced fluorescent $\mathrm{AGEs}$ by the same amount (Tx $478 \pm 26 ; p$ vs DC $10<0.001$; Tx vs D-AG $p$ ns; Fig. 4). Compared with the levels of AGEs after 6 months (DC 6), AGEs in both treatment groups were significantly elevated (D-AG and Tx, $p<0.001$ vs DC 6) (Fig.4).

Retinae from diabetic rats showed a 2.7 -fold increase in acellular capillaries after 6 months and a 4.1 fold increase after 10 months compared to agematched NC (DC 6 vs NC 6 and DC 10 vs NC 10 $p<0.001$ ). Both forms of treatment significantly reduced the progression of acellular capillaries to the same degree (D-AG 64.4 \pm 9.6 (mean \pm SEM) and Tx $71 \pm 3.9$ vs DC $101.01 \pm 7.5 ; p<0.001$ for each comparison; D-AG vs DC $651 \pm 2.1 ; p<0.05$; Tx vs DC $6 p<0.01$ ) (Fig. 5).

Determined exclusively in areas where capillary occlusions or severe irregularities were absent, chronic hyperglycaemia induced a $17.6 \%$ increase in endothelial cell numbers after 6 months (DC $62505 \pm 123$ cells $/ \mathrm{mm}^{2}$ of capillary area vs NC $62130 \pm 99$;

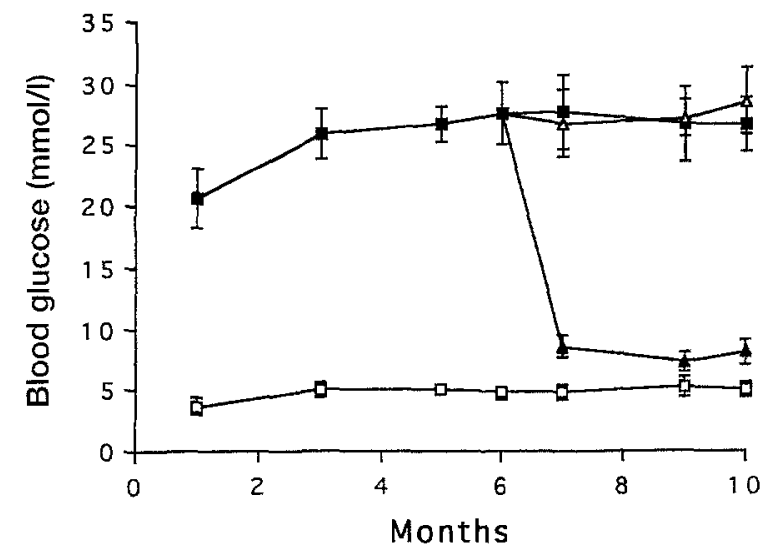

Fig. 2. Blood glucose in non-diabetic ( $\square$ ), diabetic ( $\mathbf{a})$, diabetic rats treated with aminoguanidine $(\triangle)$, and diabetic rats treated with islet transplantation $(\boldsymbol{\Delta})$. Results are shown as mean $\pm \mathrm{SD}$
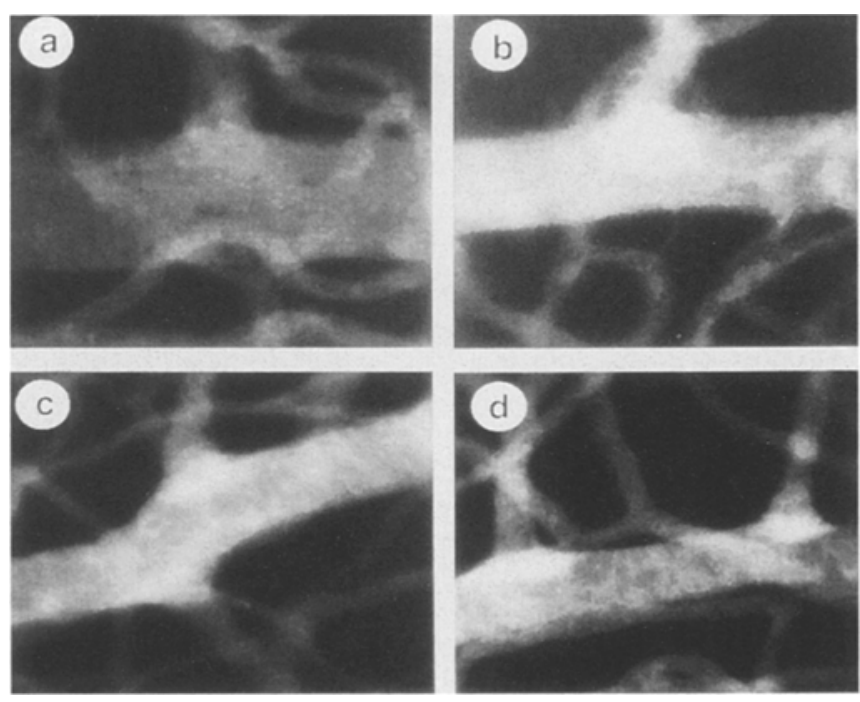

Fig. 3 a-d. Photomicrographs of retinal arteriolar autofluorescence in corresponding fields of non-diabetic (a), diabetic (b), aminoguanidine-treated diabetic (c), and diabetic rats receiving islet transplantation (d). Original magnification $400 \times$

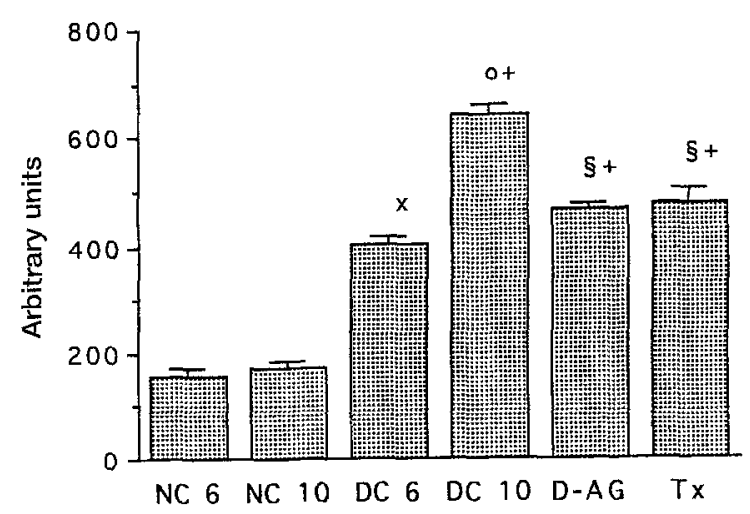

Fig.4. Retinal autofluorescence (A.U., arbitrary units) of nondiabetic, diabetic and treated diabetic rats, measured in corresponding fields of arteriolar branching sites. Results are shown as mean $\pm \mathrm{SD} .{ }^{\times} p<0.001$ vs NC $6 ;{ }^{\circ} p<0.001$ vs NC 10 ; ${ }^{+} p<0.001$ vs DC $6 ;{ }^{\$} p<0.001$ vs DC 10 


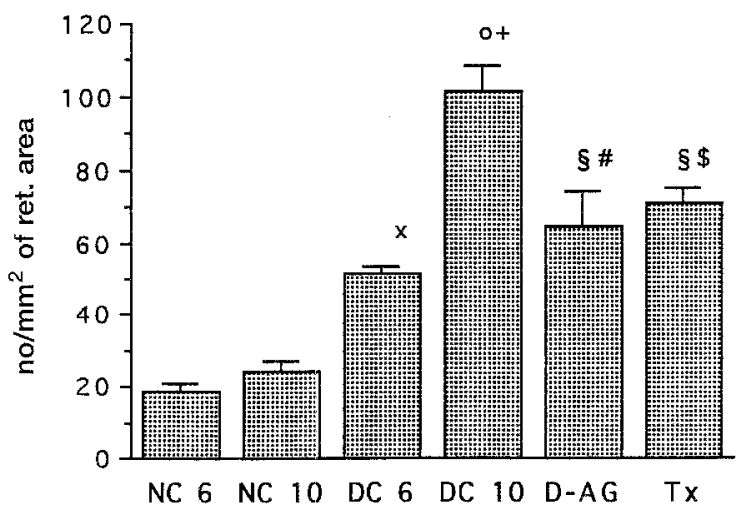

Fig. 5. Acellular capillaries of the groups studied. Results are expressed as mean \pm SEM. Additionally to symbols used in Fig. 1: ${ }^{*} p<0.05$ vs DC $6 ;{ }^{\$} p<0.01$ vs DC 6

$p<0.001$ ) and a $22.4 \%$ increase after 10 months (DC $102560 \pm 161$ cells $/ \mathrm{mm}^{2}$ vs NC $102090 \pm 115$; $p<0.001$ ). Aminoguanidine and islet Tx, both had a slight but significant effect on endothelial proliferation (D-AG 2370 1775 ; Tx $2380 \pm 119 ; p<0.05$ vs DC 10). The difference between the treatment groups and untreated diabetic rats after 6 months (DC 6) was also significant $(p<0.05)$.

The characteristic pericyte loss was $32.4 \%$ after 6 months of chronic hyperglycaemia and almost $40 \%$ after 10 months (NC $62130 \pm 80$ cells $/ \mathrm{mm}^{2}$ of capillary area vs DC $61440 \pm 165 ; p<0.001 ; \mathrm{NC} 10$ $1950 \pm 99$ vs DC $101190 \pm 118 ; p<0.001$ ). Of interest, secondary intervention with aminoguanidine and islet Tx, both completely arrested the pericyte loss (D-AG $1550 \pm 151, \quad p<0.001$ vs DC $10 ;$ Tx $1460 \pm$ $81, p<0.001$ vs DC 10). No difference existed between DC 6 and the treatment groups.

\section{Discussion}

The results from this study indicate that secondary inhibition of AGE-accumulation can retard but not arrest the progression of diabetic retinopathy in the rat model to the same degree as syngeneic islet transplantation. Concomitantly, both treatment forms were shown to retard but not arrest further accumulation of AGEs as indicated by the difference of the treated groups with the untreated diabetic group (DC 10). These data are consistent with a causal role for AGEs in the pathogenesis of diabetic retinopathy [13], and suggest that other hyperglycaemia-induced mechanisms [14] inhibited by islet Tx may be less important.

The mechanism by which aminoguanidine and islet Tx exert the beneficial effect on the retinal microvasculature appears to be by lowering the levels of AGEs [15] or their reactive precursors [16] either directly (aminoguanidine) or indirectly through the reconstitution of near-normoglycaemia (islet Tx). Our study also confirms that AGEs that have formed cannot be removed by secondary inhibition.

Continued progression of diabetic retinopathy after correction of hyperglycaemia or inhibition of AGE formation is consistent with previous descriptions of hyperglycaemic memory $[11,17]$. While the biochemical basis has not been elucidated, it is consistent with persistent damage by preformed AGEs.

Similar data of secondary aminoguanidine treatment in diabetic nephropathy have recently been reported by Soulis-Liparota et al. [18]. In their experimental design, rats were untreated for 16 weeks and treated for the same time period in comparison to normal and diabetic controls. Urinary albumin excretion was intermediate between the normal and the untreated diabetic group. In contrast to our findings, diabetes-related increase in glomerular and tubular fluorescence was completely prevented in the treated group. The measurement of autofluorescence grossly underestimates the total amounts of AGEs formed, at least in the kidney, since the majority of AGEs is non-fluorescent [19]. Therefore, non-fluorescent AGEs could have contributed to the incomplete suppression of albuminuria in these animals.

Several other properties of aminoguanidine have been described to explain its beneficial effects on diabetic vascular complications such as the inhibition of the polyol pathway or the correction of the vascular flow by acting as an inhibitor of the inducible form of the nitric oxide synthase (iNOS) [20, 21]. However, at concentrations used in our study, aminoguanidine does not have inhibitory effects on the polyol pathway or the iNOS.

In conclusion, secondary intervention of experimental diabetic retinopathy by AGE inhibition is likely to have a beneficial effect on disease progression. Concomitant retardation of retinal autofluorescence and capillary occlusions, accompanied by a preventive effect on pericyte loss, equally obtained by aminoguanidine and syngeneic islet transplantation, provides further insight into the pathogenesis of diabetic retinopathy. These data provide the basis for investigating the effect of aminoguanidine in a secondary intervention trial in patients.

Acknowledgements. This study was supported by grant $\mathrm{Ha}$ 1755/1-2 from the German Research Council, Bonn-Bad Godesberg, Germany.

\section{References}

1. Klein R, Klein BEK, Moss S et al. (1984) The Wisconsin Epidemiologic Study of Diabetic Retinopathy. II. Prevalence and risk of diabetic retinopathy when age at diagnosis is less than 30 years. Arch Ophthalmol 102: 520-526

2. Klein R, Klein BEK, Moss S et al. (1984) The Wisconsin Epidemiologic Study of Diabetic Retinopathy. III. Prevalence and risk of diabetic retinopathy when age at diagnosis is 30 or more years. Arch Ophthalmol 102: 527-532 
3. Kohner EM, Porta M, Hyer SL (1991) Pathogenesis of diabetic retinopathy and cataract. In: Pickup J, Williams G (eds) Textbook of diabetes. Blackwell Scientific Publications, Oxford, pp 564-574

4. Merimee TJ (1990) Diabetic retinopathy. A synthesis of perspectives. New Engl J Med 322:978-983

5. Engerman R, Bloodworth JMB, Nelson S (1977) Relationship of microvascular disease in diabetes to metabolic control. Diabetes 26: 760-769

6. DCCT Research Group (1993) The effect of intensive treatment of diabetes on the development and progression of long-term complications in insulin-dependent diabetes mellitus. N Engl J Med 329: 977-986

7. Hammes HP, Martin S, Federlin K et al. (1991) Aminoguanidine treatment inhibits the development of experimental diabetic retinopathy. Proc Nat Acad Sci USA 88:1155511558

8. Hammes HP, Brownlee M, Edelstein D et al. (1994) Aminoguanidine inhibits the development of accelerated diabetic retinopathy in the spontaneous hypertensive rat. Diabetologia 37: 32-35

9. Lacy PE, Kostianovsky YM (1967) Method for the isolation of intact islets of Langerhans from the rat pancreas. Diabetes 16: 35-39

10. Kuwabara T, Cogan DG (1960) Studies of retinal vascular patterns. I. Normal architecture. Arch Ophthalmol 64: 904-911

11. Engerman RL, Kern TS (1987) Progression of diabetic retinopathy during good glycemic control. Diabetes 36 : $808-$ 812

12. Beaven MA, Gordon JW, Jacobson S, Sever W (1969) A specific and sensitive assay for aminoguanidine: its applica- tion to a study of the disposition of aminoguanidine in animal tissues. J Pharmacol Exp Ther 165: 14-22

13. Brownlee M (1994) Lilly lecture 1993. Glycation and diabetic complications. Diabetes 43: 836-841

14. Ruderman NB, Williamson JR, Brownlee M (1992) Glucose and diabetic vascular disease. FASEB J 6:2905-2914

15. Nicholls K, Mandel TE (1989) Advanced glycosylation end-products in experimental murine diabetic nephropathy: effect of islet isografting and aminoguanidine. Lab Invest 60: 486-493

16. Fu MX, Wells-Knecht KJ, Blackledge JA et al. (1994) Glycation, glycoxidation, and cross-linking of collagen by glucose. Kinetics, mechanisms, and inhibition of late stages of the Maillard reaction. Diabetes 43: 676-683

17. Roy S, Sala R, Cagliero E, Lorenzi M (1990) Overexpression of fibronectin induced by diabetes or high glucose: phenomenon with a memory. Proc Nat Acad Sci USA 87: 404-408

18. Soulis-Liparota T, Cooper M, Jerums G (1993) Effects of early and late aminoguanidine therapy on experimental diabetic nephropathy. 5th Int Symp on Maillard Reaction, Minnesota, USA (Abstract)

19. Mitsuhashi T, Nakayama H, Itch S, Kuwajima S et al. (1993) Immunochemical detection of advanced glycation end products in renal cortex from STZ induced diabetic rats. Diabetes 42: 826-833

20. Kumari K, Umar S, Bansal V, Sahib MK (1991) Monoaminoguanidine inhibits aldose reductase. Biochem Pharmacol 41: 1527-1528

21. Corbett JA, Tilton RG, Chang K et al. (1992) Aminoguanidine, a novel inhibitor of nitric oxide formation, prevents diabetic vascular dysfunction. Diabetes 41: 552-556 